\title{
Spectropolarimetry of SN 2006aj at 9.6 days ${ }^{\star}$
}

\author{
J. R. Maund ${ }^{1}$, J. C. Wheeler ${ }^{1}$, F. Patat ${ }^{2}$, D. Baade ${ }^{2}$, L. Wang ${ }^{3}$, and P. Höflich ${ }^{4}$ \\ 1 Dept. of Astronomy and McDonald Observatory, The University of Texas at Austin, 1 University Station, C1400, Austin, \\ Texas 78712-0259, USA \\ e-mail: jrmaastro.as.utexas.edu \\ 2 ESO - European Organisation for Astronomical Research in the Southern Hemisphere, Karl-Schwarzschild-Str. 2, \\ 85748 Garching b. München, Germany \\ 3 Department of Physics, Texas A\&M University, College Station, Texas 77843-4242, USA \\ 4 Department of Physics, Florida State University, Tallahassee, Florida 32306-4350, USA
}

Received 18 July 2007 / Accepted 25 August 2007

\section{ABSTRACT}

Context. Spectropolarimetry has been used to directly measure the asymmetries of Supernovae (SNe), Gamma-Ray Bursts (GRBs) and X-Ray Flashes (XRFs).

Aims. We determine whether non-axial asymmetries are present in SNe that are associated with GRBs and XRFs, given the particular alignment of the jet axis and axis of symmetry with the line of sight in these cases.

Methods. We performed spectropolarimetry with the Very Large Telescope (VLT) FORS1 instrument of the type Ic SN 2006aj, associated with the XRF 060218, at $V$-band maximum at 9.6 rest frame days after the detection of the XRF. Due to observations at only 3 retarder plate angles, the data were reduced assuming that the instrumental signature correction for the $U$ Stokes parameter was identical to the correction measured for $Q$.

Results. We find SN 2006aj to be highly polarized at wavelengths corresponding to the absorption minima of certain spectral lines, which are particularly strong for O I $7774 \AA$ and Fe II, observed at $4200 \AA$ with a polarization of 3\%. The value of the Interstellar Polarization is not well constrained by these observations and, considering the low polarization observed between $6000-6500 \AA$, the global asymmetry of the $\mathrm{SN}$ is $\lesssim 15 \%$. O I and Fe II lines share the same location in the $Q-U$ plane, that differs from Ca II. Conclusions. SN 2006aj exhibits a higher degree of line polarization than other SNe associated with GRBs and XRFs. The polarization associated with spectral lines implies significant asymmetries of these elements with respect to each other and to the line of sight. This is contrary to the standard picture of SNe associated with GRBs/XRFs, where the axis of symmetry of the SN is aligned with the GRB jet axis and the line of sight.

Key words. supernovae: general - supernovae: individual: SN 2006aj - supernovae: individual: SN 2002ap supernovae: individual: SN 2003dh - techniques: polarimetric

\section{Introduction}

The number of observed core-collapse Supernovae (CCSNe) associated with Gamma Ray Bursts (GRBs) and X-ray Flashes (XRFs) is still low and there are few examples of such $\mathrm{SNe}$ which have also been observed at early times with spectropolarimetry: SN 1998bw (GRB 980425; Patat et al. 2001) and SN 2003dh (GRB 030329; Kawabata et al. 2003). Here we present spectropolarimetry of the type Ic SN 2006aj associated with XRF 060218.

XRF 060218 was detected on 2006 Feb. 18.15 UT by the Swift Burst Alert Telescope (Cusumano et al. 2006a), and located at $\alpha_{\mathrm{J} 2000}=03^{\mathrm{h}} 21^{\mathrm{m}} 39.7^{\mathrm{s}}$ and $\delta_{\mathrm{J} 2000}=+16^{\circ} 52^{\prime} 01.8^{\prime \prime}$ by the Swift X-ray Telescope (Cusumano et al. 2006b). The XRF had a long duration ( $3000 \mathrm{~s}$; Campana et al. 2006), and was reported as being under-luminous and softer than normal GRBs (Pian et al. 2006). Mirabal \& Halpern (2006) reported optical spectroscopy of the afterglow, at Feb. 20.04, revealing nebular emission features from the host galaxy with $z=0.033$. Fatkhullin et al. (2006) commented that optical spectroscopy of the afterglow revealed the presence of a type Ic SN similar to

* Based on observations made with ESO Telescopes at the Paranal Observatory, under programme 76.D-0177(A).
SN 1997ef, and this SN was subsequently labelled SN 2006aj (Soderberg et al. 2006a). Pian et al. (2006) and Modjaz et al. (2006) observed SN 2006aj to have a rapid rise to $V$-band maximum in 10 days. Gorosabel et al. (2006) observed evolution in the $R$-band linear polarization, from a rapidly evolving high polarization $(\sim 4.5 \%$ at 3 days following the XRF) and a lower polarization $(\sim 1.4 \%)$ after the $V$-band maximum of the SN light curve. Late-time spectropolarimetry of SN 2006aj (at 206 rest frame days after the XRF) showed no polarization to $2 \%$ at $3 \sigma$, while observations of the nebular spectrum revealed asymmetric line profiles for [OI] $\lambda \lambda 6300,6363$ (Mazzali et al. 2007).

\section{Observations}

Spectropolarimetry of SN 2006aj was acquired on 2006 Feb. 28.02, using the European Southern Observatory (ESO) Very Large Telescope (VLT) FOcal Reducer and low dispersion Spectrograph (FORS1) instrument in the spectropolarimetry "PMOS" mode (Appenzeller et al. 1998). This corresponds to 9.87 days following the original detection of the XRF, and 9.55 days in the rest frame. At this epoch, SN 2006aj was approaching the solar position, limiting the available observing time. Due to this constraint, a full spectropolarimetry dataset was 
not acquired and, due to the nature of spectropolarimetry observations, this dataset could not be completed at a later epoch. Observations of SN 2006aj were acquired with the retarder plate at only three positions: $0.0,45^{\circ} .0$ and $22^{\circ} .5$. Exposure times were $900 \mathrm{~s}$, with the retarder plate positioned at 0.0 and $45^{\circ} .0$, and $793 \mathrm{~s}$, at $22^{\circ} .5$. The G300V grism was used, with a pixel scale $2.6 \AA \mathrm{px}^{-1}$ and spectral resolution, measured from arc lamp calibration spectra, of $12.6 \AA$. The data were reduced in the standard manner, following the routine outlined by Maund et al. (2007a). Due to incomplete observations, the Stokes parameters were determined following the technique of Maund (2007), which assumes that the "instrumental signature corrections" of the $Q$ and $U$ Stokes parameters, $\epsilon_{Q}$ and $\epsilon_{U}$, are approximately the same.

Given the broadness of the spectroscopic features, due to high expansion velocities ( $\gtrsim \sim 18000 \mathrm{~km} \mathrm{~s}^{-1}$; Pian et al. 2006), the Stokes parameters were coarsely re-sampled to $50 \AA$ wavelength bins. A correction for the recessional velocity of the host galaxy was applied to the data, using $z=0.033$ derived from the narrow nebular lines observed in this data (this is consistent with previously reported measures of the recessional velocity; e.g. Mirabal \& Halpern 2006).

\section{Results and analysis}

The observed Stokes parameters and flux spectrum of SN 2006aj at 9.55 days are presented as Fig. 1. This observation was acquired at the maximum of the $V$-band $\left(m_{V}=17.5\right.$; Modjaz et al. 2006) and bolometric light curves of the SN (Gorosabel et al. 2006; Pian et al. 2006). The flux spectrum is composed of a series of broad overlapping absorption and emission features; the flux spectrum has been extensively discussed by Pian et al. (2006) and Modjaz et al. (2006). The polarization spectrum is observed to be variable over the wavelength range of 1 bin. The wavelength range of 6000-6500 $\AA$ is characterised by a low level of polarization of $0.5 \pm 0.3 \%$ (null polarization at a $2 \sigma$ level). Over the larger range of $4500-6500 \AA$, the polarization is $\$ 2 \%$. The highest degrees of polarization are $2-4 \%$ and are associated with features at the blue and the red extreme of the spectrum. A wavelength-dependent Interstellar Polarization (ISP) component may be responsible for some of this polarization, and is discussed later, but it is clear that significant variability of the polarization spectrum over small wavelength ranges implies that there is significant polarization intrinsic to SN 2006aj itself. In addition, we note that the bulk of the polarization signature derives from the $U$ Stokes parameter, but the features are not correlated with the $Q$-instrumental signature correction.

The $R$-band polarimetry of Gorosabel et al. (2006), at 13 days after the XRF, is $2 \sigma$ higher than the spectropolarimetry of three days prior (see Fig. 1). There is some variability in the degree of polarization across the observed wavelength range of the $R$-band, but there appears to be no obvious wavelength dependence that would contribute to the broad-band measurement of Gorosabel et al. A region of higher polarization is observed immediately redward of $7000 \AA$ in the rest frame and would have contributed to the $R$-band polarization if the recessional velocity of the host galaxy were lower. Differences between the polarization and the polarization angle of the spectropolarimetry and the observations of Gorosabel et al. (2006) are most likely due to the differences in the epoch at which the respective observations were conducted.

The rise in the polarization at the red extreme of the spectrum is most likely associated with O I $\lambda 7774$ and the Ca II IR triplet.
There are peaks in the polarization spectrum which, given the correspondence of polarization peaks and absorption minima of P Cygni profiles in SN spectra (McCall 1984), can be associated with the absorption minima of these lines. The polarization peaks correspond to absorption velocities of O $\lambda 7774$ at $29200 \pm$ $2000 \mathrm{~km} \mathrm{~s}^{-1}$ and the Ca II IR triplet $25800 \pm 1800 \mathrm{~km} \mathrm{~s}^{-1}$. The large uncertainties on these velocities arise from the coarse rebinning of the Stokes parameters to $50 \AA$ and line blending. At the blue end of the spectrum, the polarization of $4 \pm 0.7 \%$ at $4200 \AA$ is associated with Fe II multiplets 37 and 38 at $20500 \pm$ $3500 \mathrm{~km} \mathrm{~s}^{-1}$. We suggest that peaks in the polarization between 4500-5000 $\AA$ are due to redder Fe II lines, particularly that of Fe II $\lambda 5169$ at $4850 \AA$, implying a velocity of $18500 \pm$ $3000 \mathrm{~km} \mathrm{~s}^{-1}$. The lower polarization of the redder Fe II lines is not unexpected, as the bluer Fe II lines of multiplets 37 and 38 have been observed to produce a single P Cygni profile, given the small difference in wavelength of the constituent lines and the high velocities observed in $\mathrm{SNe}$, whereas the redder lines can be resolved (Maund et al. 2007a). We note that these velocities are similar to the values quoted by Pian et al. (2006) for the photospheric velocity, measured with the Fe lines, and Mazzali et al. (2006), who consider an O-dominated shell of ejecta at $20000 \lesssim v \lesssim 30000 \mathrm{~km} \mathrm{~s}^{-1}$. There are two minor peaks in polarization between $5500 \AA<\lambda<6000 \AA$ which may be associated with Si II $\lambda 6355$, that Mazzali et al. (2006) identified in spectra.

There is an additional apparent peak in the polarization spectrum at $7840 \AA$. The measured polarization, at this wavelength, has a high uncertainty, because it is coincident with a low $\mathrm{S} / \mathrm{N}$ portion of the spectrum at a broad telluric absorption. This feature is not, therefore, intrinsic to the $\mathrm{SN}$, nor significantly more polarized than the immediately surrounding wavelengths.

The observed Stokes parameters are plotted on the $Q-U$ plane in Fig. 2. The data conform to an obvious dominant axis, which is elongated along the $U$ axis. Some degree of elongation along the $U$ axis is to be expected, since the errors on the $U$ Stokes parameter are generally double those determined for $Q$. This elongation is, however, substantially larger than the uncertainties on the $U$ parameter, suggesting it is real and not just due to significant differences between $\epsilon_{Q}$ and $\epsilon_{U}$. No obvious loops are observed, corresponding to the Stokes parameters across particular spectral lines, in the $Q-U$ plane (Maund et al. 2007a). This is most likely due to the coarse rebinning and relatively low level of S/N ( 200). The Stokes parameters at wavelengths corresponding to different spectral lines are, however, observed to occupy different portions of the $Q-U$ plane. The polarization angles of the Fe II and O I features are similar and these spectral features occupy approximately the same location ( $U<0$ ) on the $Q-U$ plane (see Fig. 2). The Ca II IR triplet is distinct from the Fe II and O I features, with a polarization angle offset from Fe II and O I by $\sim 90^{\circ}$ and occupying the $U>0$ region of the $Q-U$ plane. The dominant axis is principally defined by those features with the highest polarizations, which correspond to the Fe II, O I and Ca II absorption features. The dominant axis is offset from the origin of the $Q-U$ plane, which can be due to the ISP or can be intrinsic to the SN itself (Maund et al. 2007b).

Schlegel et al. (1998) ${ }^{1}$ give the foreground Galactic reddening of $E(B-V)=0.14$, which corresponds to $p_{\text {ISP }}<$ $1.26 \%$. Mazzali et al. (2007) report late-time spectropolarimetry of SN 2006aj, with an upper limit on the total polarization of $p(3 \sigma)<2 \%$ (at late times, as the density of scattering

\footnotetext{
1 http://nedwww.ipac. caltech.edu/
} 


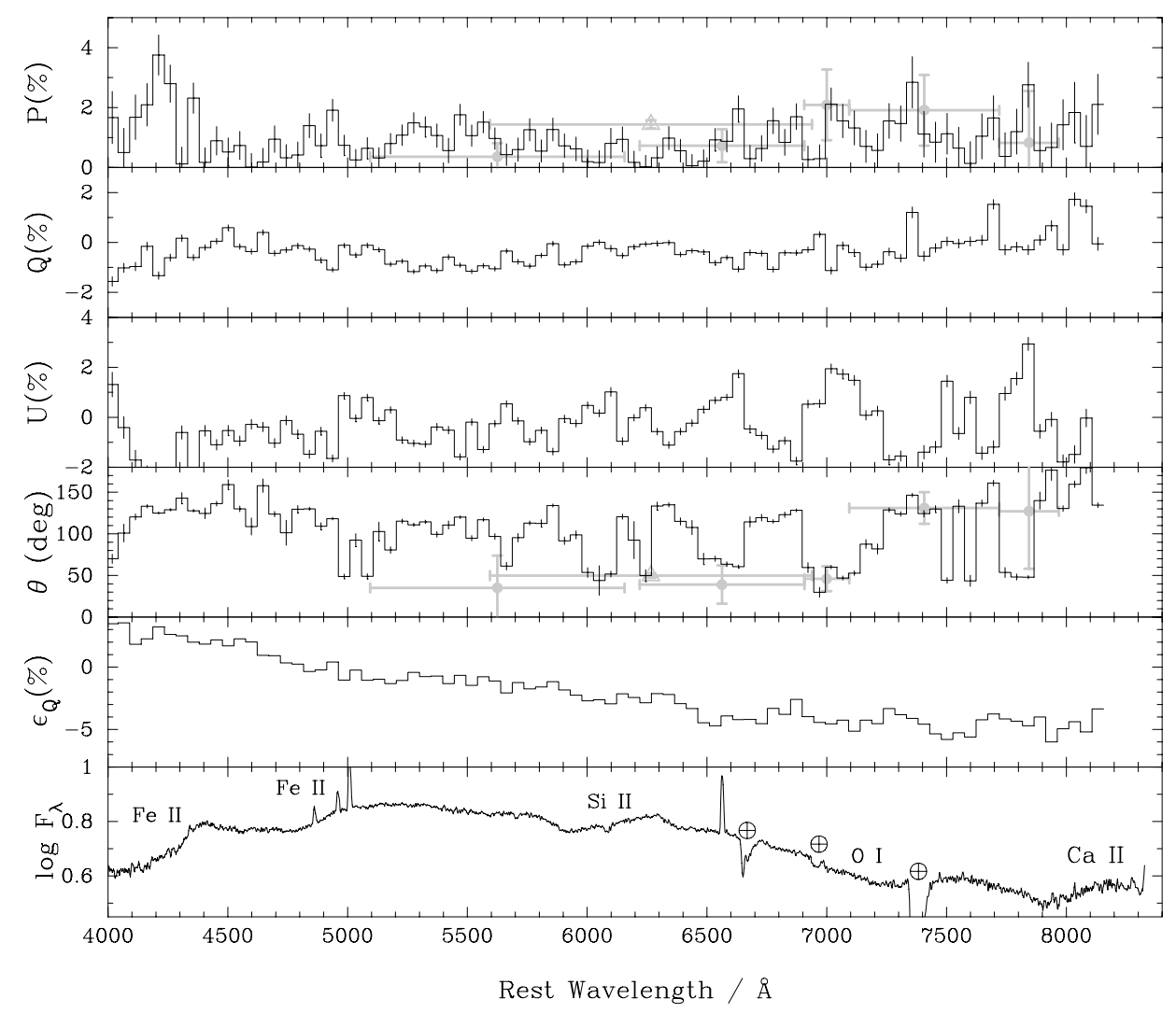

Fig. 1. Spectropolarimetry of SN 2006aj at 9.55 days. From top to bottom: total polarization $p$, the $Q$ Stokes parameters, the $U$ Stokes parameter, the polarization angle $\theta$, the instrumental signature correction $\epsilon_{Q}$ observed for the $Q$ Stokes parameters and the flux spectrum $\left(\mathrm{erg} \mathrm{s}^{-1} \mathrm{~cm}^{-2} \AA^{-1}\right)$. The wavelength scales for the polarization parameters have been rebinned to $50 \AA \mathrm{bin}^{-1}$, and the flux spectrum is binned at $2.6 \AA$ bin ${ }^{-1}$. Also shown are polarization parameters of Gorosabel et al. (2006) $(\triangle)$ for SN 2006aj at 13.7 days and Kawabata et al. (2003) (•) for SN 2003dh at 16 days after $V$-band maximum. The wavelength scales for each of the data sets have been corrected for the recessional velocities of the respective host galaxies. The narrow lines in the flux spectrum (bottom panel) are nebular lines arising in the host galaxy. Telluric absorption features are indicated by $\oplus$.

particles decreases $p \rightarrow p_{\text {ISP), while Gorosabel et al. (2006) sug- }}$ gest that the observed $R$-band polarization of $1.4 \%$ at days $>13$ is consistent with $E(B-V)>0.15$. We note that the data in the wavelength range of 6000-6500 $\AA$ suggests $E(B-V)>0.05$, for no intrinsic polarization or ISP depolarization.

There is a peak in the polarization at $5500 \AA$, which is not matched by an obvious absorption feature in the flux spectrum. This may well represent the maximum of the ISP, where $p=$ $1.4 \pm 0.4 \%$, which would be consistent with the ISP estimated by Gorosabel et al.

\section{Discussion and conclusions}

It is particularly important to determine whether the assumption that $\epsilon_{U} \approx \epsilon_{Q}$ is correct. It is clear from comparison with the data of Gorosabel et al. (2006) that this assumption is correct to within $\sim 1 \%$. Examination of the three exposures, at each of the retarder plate positions, suggests that the observations were not in the regimes identified by Maund (2007) for which $\epsilon_{Q}$ and $\epsilon_{U}$ are expected to diverge: large gain gradient between the ordinary and extraordinary rays and polarizations $\gtrsim 20 \%$. Monte Carlo models of the observations, given a signal-to-noise ratio of $\sim 200$ at each retarder plate angle, yielded an error on the $U$ instrumental signature correction $\Delta \epsilon_{U} \sim \pm 0.3 \%$. This approach is limited, however, as any defects peculiar to the observation with the retarder plate at 22.5 cannot be identified, as we did not obtain an observation at $67^{\circ} .5$. As the Stokes parameters are calculated using normalised flux differences, the corrections are also flux normalized; the difference in exposures times leads to an increase in the uncertainty on $U$ due to Poisson noise as well as $\Delta\left(\epsilon_{Q}-\epsilon_{U}\right)$.

In the ESO archive there are seven spectropolarimetry datasets of SNe in the 19 days prior to XRF 060218/SN 2006aj and two sets in the month following (Patat et al. 2007) which, with complete observations at all four retarder plate positions, give errors on the $U$ instrumental signature corrections, $\epsilon_{U}$, consistent with the signal-to-noise and the model presented by Maund (2007). These observations show no obvious time dependent defects over the time period immediately surrounding this observation of SN 2006aj. The caveat that the Stokes parameters were not determined completely independently must always be remembered when considering these results.

The wavelength region 5500-6500 $\AA$ shows the smallest degree of polarization variability in the polarization spectrum, where the $p \sim 0.45 \%$. A similar polarization spectrum was observed for SN 1998bw/GRB 980425, with little wavelength dependence across the same wavelength range (for observations at 7 days before and 10 days after $V$ maximum, although the level of polarization was lower at the later epoch; Patat et al. 2001). The polarization across this wavelength range can be used to place an upper limit on the degree of global asymmetry of SN 2006aj, assuming that all the polarization is intrinsic to the SN. Comparison with Hoflich (1991) shows that this level of polarization requires deviations from a spherical symmetry of 
$\$ 15 \%$. In the presence of a substantial ISP, such as that discussed by Gorosabel et al. (2006), the degree of the asymmetry of the photosphere would tend to zero. Alternatively, if the polarization angle of the ISP is fortuitously counter aligned with the polarization angle of the intrinsic SN polarization, then the ISP would effectively depolarize the SN. This would yield an underestimate for the asymmetry of SN 2006aj. Regardless of the ISP, however, the wavelength-dependence of the polarization, especially coincident with absorption features in the flux spectrum, is not consistent with a smoothly varying ISP law. This demonstrates that asymmetries are significantly present in SN 2006aj.

The high degree of polarization associated with O I $\lambda 7774$ and the Ca II IR triplet in the data of SN 2006aj is similar to that observed in high-velocity $\mathrm{SNe}$ and other $\mathrm{SNe}$ associated with GRBs, such as SN 2003dh/GRB 030329. For SN 2003dh there is a clear rise in polarization at the red end of the spectrum associated with the approximate location of the O I $\lambda 7774$ line, as shown in Fig. 1. The data of Kawabata et al. (2003) was, however, acquired at $\sim 16$ days after the corresponding $V$-maximum (18 days after the GRB 030329; Matheson et al. 2003). The wavelength range containing the Fe II lines was not covered with the spectropolarimetry observations of SN 1998bw and SN 2003dh. The Fe II lines were observed in SN 2002ap and were less polarized, at $0.3 \%$ at $V$-maximum, than those observed in SN 2006aj, and substantially less polarized than the corresponding O I $7774 \AA$ line at 1\% (Wang et al. 2003). The Ca II IR triplet in SN 2006aj is also more polarized than observed in SN 2003dh (Kawabata et al. 2003).

SN 2006aj does not show conspicuously higher polarization than other examples of CCSNe. Some "stripped-core" CCSNe show higher levels of polarization of their core layers, implying higher degrees of asymmetry, over larger wavelength ranges than observed for SN 2006aj (Maund et al. 2007b). The highest polarization observed for a type Ic SN was $\gtrsim 4 \%$ for SN 1997X (Wang et al. 2001). In the cases of future, better observed CCSNe associated with GRBs, for which the ISP can be more thoroughly measured, the intrinsic polarizations and polarization angles may have similarities given the preferred orientation of such objects.

The polarization angles of the Fe II and Ca II lines are significantly different, and subtraction of an ISP, with constant polarization angle, would change the absolute values of the polarization angles of these features, but would not eliminate their relative offset. The dissimilar polarization angles is suggestive of different geometries of these species within the ejecta. Mazzali et al. (2007) note that spectral synthesis models of the SN 2006aj suggest that the $\mathrm{O} \mathrm{I}$, in the late time ejecta, is configured in a face-on disk, while Ni has an almost spherical geometry. Neither of these geometries provides the distinct non-axisymmetric component which would yield such high levels of polarization.

The presence of strong polarization associated with lines points to an asymmetric distribution of these elements with respect to the angle at which SN 2006aj is observed. Importantly, SN 2006aj was observed with a specific orientation, with the line of sight and the jet axis being coincident. Pian et al. (2006) rule out the scenario of XRF 060218 having been observed significantly off-axis, such that an axisymmetric SN would appear polarized due to inclination effects. If the jet axis defines the axial symmetry, then the presence of asymmetry observed as polarization is difficult to rectify with the axisymmetric standard model of the SNe-GRBs (Woosley \& Bloom 2006). Alternatively, if the jet opening angle is large (e.g. $\theta_{\mathrm{j}}>80^{\circ}$ measured by Soderberg et al. (2006b) from the radio light curve of SN 2006aj) the line

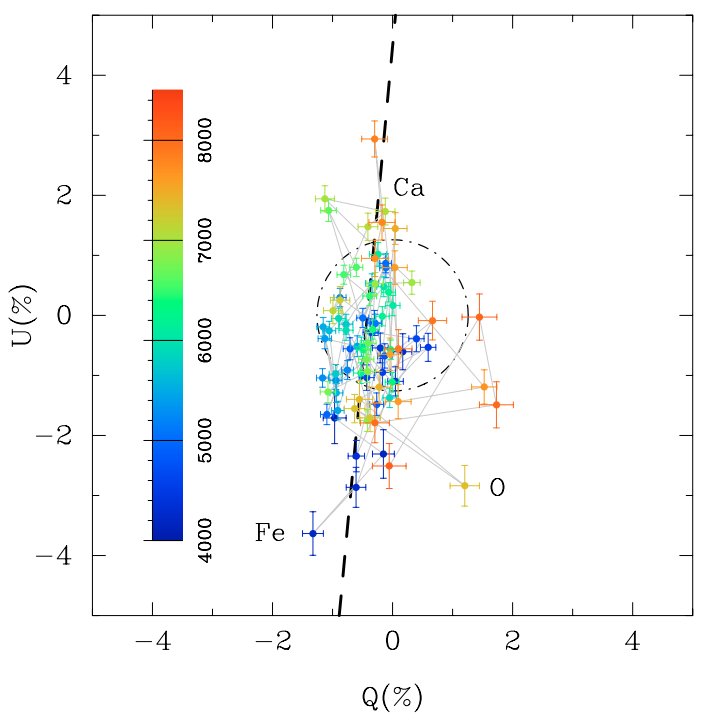

Fig. 2. The observed Stokes parameters of SN 2006aj, on the $Q-U$ plane. The data have been rebinned to $50 \AA$, but have not been corrected for an ISP component. The maximum ISP $p<1.26 \%$, corresponding to the foreground Galactic extinction of $E(B-V)=0.14$, is indicated by the dot-dashed circle. The points are colour-coded by wavelength, according to the scheme of the colour bar. The heavy dashed line indicates the dominant axis of the data (Wang et al. 2001).

of sight may be significanty inclined with respect to the expected axis of symmetry of the progenitor and the SN, but still allow the prompt emission to be observed.

The wavelength-dependent polarization observed, at later epochs, for SN 2003dh implies that the presence of such asymmetries is not limited to SN 2006aj, and is a property of SNe associated with GRBs.

\section{References}

Appenzeller, I., Fricke, K., Furtig, W., et al. 1998, The Messenger, 94, 1 Campana, S., Mangano, V., Blustin, A. J., et al. 2006, Nature, 442, 1008 Cusumano, G., Barthelmy, S., Gehrels, N., et al. 2006a, GRB Coordinates Network, 4775, 1

Cusumano, G., Moretti, A., Tagliaferri, G., Kennea, J., \& Burrows, D. 2006b, GRB Coordinates Network, 4786, 1

Fatkhullin, T. A., Sokolov, V. V., Moiseev, A. V., Guziy, S., \& Castro-Tirado, A. J. 2006, GRB Coordinates Network, 4809, 1

Gorosabel, J., Larionov, V., Castro-Tirado, A. J., et al. 2006, A\&A, 459, L33 Hoflich, P. 1991, A\&A, 246, 481

Kawabata, K. S., Deng, J., Wang, L., et al. 2003, ApJ, 593, L19

Matheson, T., Garnavich, P. M., Stanek, K. Z., et al. 2003, ApJ, 599, 394

Maund, J. 2007, A\&A, submitted

Maund, J., Wheeler, J., Patat, F., et al. 2007a, MNRAS, accepted

Maund, J., Wheeler, J., Patat, F., et al. 2007b, ApJ, accepted

Mazzali, P. A., Deng, J., Nomoto, K., et al. 2006, Nature, 442, 1018

Mazzali, P. A., Foley, R. J., Deng, J., et al. 2007, ApJ, 661, 892

McCall, M. L. 1984, MNRAS, 210, 829

Mirabal, N., \& Halpern, J. P. 2006, GRB Coordinates Network, 4792,

Modjaz, M., Stanek, K. Z., Garnavich, P. M., et al. 2006, ApJ, 645, L21

Patat, F., Cappellaro, E., Danziger, J., et al. 2001, ApJ, 555, 900

Patat, F., Baade, D., Höflich, P. A., et al. 2007, A\&A, in press

Pian, E., Mazzali, P. A., Masetti, N., et al. 2006, Nature, 442, 1011

Schlegel, D. J., Finkbeiner, D. P., \& Davis, M. 1998, ApJ, 500, 525

Soderberg, A., Berger, E., \& Schmidt, B. 2006a, IAU Circ., 8674, 2

Soderberg, A. M., Kulkarni, S. R., Nakar, E., et al. 2006b, Nature, 442, 1014

Wang, L., Howell, D. A., Höflich, P., \& Wheeler, J. C. 2001, ApJ, 550, 1030

Wang, L., Baade, D., Höflich, P., \& Wheeler, J. C. 2003, ApJ, 592, 457

Woosley, S. E., \& Bloom, J. S. 2006, ARA\&A, 44, 507 